\title{
Bypass anillado ¿cuál es su real utilidad? Estudio comparativo a diez años*
}

\author{
Drs. WILLIAM AWAD F. ${ }^{1,2,3,5}$, ÁLVARO GARAY M. ${ }^{2,3,5}$, \\ CRISTIÁN MARTÍNEZ M..$^{3,4,5}$, JULIO YARMUCH G. ${ }^{1,5}$ \\ 1 Departamento de Cirugía Hospital Clínico Universidad de Chile. \\ 2 Servicio de Cirugía Hospital San Juan de Dios. \\ 3 Clínica Las Lilas. \\ 4 Servicio de Cirugía Hospital Sótero del Río. \\ 5 Integramédica. \\ Santiago, Chile.
}

\begin{abstract}
Banded gastric bypass. A ten years evaluation

Background: Placing a ring around the gastric reservoir could improve the weight lowering effects of gastric bypass. Previous studies have shown positive results of banded gastric bypass. Aim: To evaluate the long-term outcome of patients subjected to banded gastric bypass (GB) and non-banded GB procedures. Material and Methods: Ten years follow up of 260 non randomized obese patients who underwent banded GB and 218 patients in whom the ring was not placed. Excess weight loss (EWL), quality of life (QOL), food tolerance (FT), and correction of comorbidities were assessed. Results: The percentage EWL at ten years of follow up was 82 and $63 \%$ among patients subjected to banded and non-banded gastric bypass, respectively $(\mathrm{p}<0.01)$. In three patients with banded GB, there was a migration of the ring to the interior of the stomach. Stenosis of the gastro-jejuno anastomosis occurred in 4 and $0.4 \%$ of patients subjected to banded and nonbanded GB Perception of quality of life was similar in both groups. The outcome in terms of comorbidities was not conclusive. Conclusions: There is a clear advantage in terms of EWL among patients subjected to banded GB. No differences in quality of life were found in both groups.
\end{abstract}

Key words: Banded bypass, weight loss, bariatric surgery.

\section{Resumen}

Introducción: Muchos cirujanos han procurado mejorar los resultados del bypass gástrico agregando algún mecanismo restrictivo, como es la instalación de un anillo alrededor del bolsillo. Estudios previos han mostrado resultados positivos con el bypass gástrico anillado (BPGA), sin embargo, no hay estudios comparativos con resultados en el largo plazo para establecer la real utilidad de agregar un anillo durante la cirugía del bypass gástrico. Objetivo: Este estudio está dirigido a comparar el resultado a largo plazo entre el bypass gástrico (BPG) y el bypass gástrico anillado (BPGA). Material y Método: Estudiamos 260 pacientes obesos

*Recibido el 11 de noviembre de 2011 y aceptado para publicación el 9 de enero de 2012.

Los autores no declaran conflictos de interés.

Correspondencia: Dr. William Awad F.

Santos Dumont 999, Santiago, Chile. Fax: 562-6780884.

williamawad@obesidadycirugia.cl 
operados con anillo y 218 sin anillo. Fueron seguidos por 10 años, y se investigaron la pérdida de exceso de peso (PEP), la calidad de vida (CDV), tolerancia a la alimentación, y resolución de comorbilidades. Resultados: Hay una clara diferencia en el \% PEP desde el tercer año de control; alcanza a un 19\% de diferencia en favor del BPGA al término del estudio, cifra altamente significativa. El anillo disminuye en parte la facilidad de ingesta pero esto no es sentido por los pacientes como una disminución de la calidad de vida (CDV). Los resultados en cuanto a comorbilidades no son concluyentes. Conclusiones: Hay una clara diferencia en el resultado en cuanto a PEP. La calidad de vida es similar en ambos grupos. Es importante seleccionar el material adecuado y el correcto tamaño del anillo para mejorar los resultados y evitar complicaciones.

Palabras clave: Bypass gástrico anillado, calidad de vida, pérdida de exceso de peso.

\section{Introducción}

Ha habido muchos intentos de modificación del bypass gástrico, especialmente para reducir la re-ganancia de peso ${ }^{1}$. La idea de restringir el paso del alimento a través de la gastroenteroanastomosis está seguramente basada en la técnica de efectuar gastroplastías. Inicialmente se hacían sin restricción pero los resultados fueron bastante pobres, y rápidamente se empezó a agregar un refuerzo alrededor del tracto de salida del bolsillo gástrico, lo que mejoró considerablemente los resultados en la PEP. Las primeras gastroplastías eran transversas y se reforzaban con una sutura corrida no absorbible sobre la línea de grapas. Sin embargo, se consiguieron bastante mejores resultados con la gastroplastía vertical en curva menor, que por tener una pared más firme se dilata menos que el bolsillo transverso o de la curva mayor. Además, Mason, agregó el refuerzo con anillo que se ponía alrededor del bolsillo gástrico. Este procedimiento es sin duda el precursor del bolsillo gástrico que se hace en el bypass anillado. En nuestra experiencia con la gastroplastía, a comienzos de los 80, hicimos la gastroplastía transversa sin refuerzo, pero pronto cambiamos a la técnica vertical anillada que mejoró los pobres resultados del procedimiento anterior, pues reducía el riesgo de dilatación del tracto de salida del bolsillo, con lo que se reducía así la recuperación del peso $^{2-4}$.

Alrededor del año 1976, Fobi desarrolló su forma de hacer el bolsillo gástrico en el bypass, el llamado Fobi pouch, que era un bolsillo vertical, con el asa enteral interpuesta entre ambas partes del estómago, y un anillo alrededor del bolsillo, que quedaba fijo por esta interposición de asas. Al mismo tiempo, Capella llegó a la misma conclusión y mismo diseño, pasando por etapas de la gastroplastía vertical, a ponerle un anillo y luego a la sección del estómago conformando la técnica de BPGA como la conocemos hoy, popularizándola en Latinoamérica ${ }^{4,5}$.

Linner fue el primero en poner una banda en el bypass gástrico. Fobi relató en el III Congreso de IFSO Latinoamericano, que cuando inició esta práctica ponía el anillo en la misma anastomosis y tuvo numerosas complicaciones especialmente migraciones al interior del estómago. A raíz de esto, decidió poner un anillo de silastic al menos a un centímetro sobre la anastomosis con lo cual prácticamente no volvió a tener migraciones.

No hay muchas publicaciones que comparen resultados a largo plazo del BPG con y sin anillo.

En este estudio, prospectivo no aleatorizado, $\mathrm{y}$ que es la continuación de un estudio previo, comparamos el resultado alejado a 10 años de seguimiento, de los siguientes parámetros: porcentaje de pérdida de exceso de peso (\% PEP), calidad de vida (CDV), tolerancia a la alimentación y resolución de comorbilidades, en dos grupos de pacientes sometidos a bypass gástrico con y sin anillo de restricción.

\section{Material y Método}

Doscientos cincuenta y ocho pacientes obesos mórbidos, con un índice de masa corporal (IMC) entre 35 y 39,9 , con comorbilidades, o pacientes con IMC $\geq 40$, fueron sometidos a un BPGA entre junio de 1998 y junio de 2004. (Grupo A). Al comienzo operábamos en dos clínicas. En una de ellas usamos un parche vascular de polytetrafluoretileno (PTFE), que estaba disponible en esa clínica: En la segunda clínica usamos un parche vascular GoreTex ${ }^{\circledR}$ Acuseal que es un material fino y maleable muy bien tolerado. Colocamos una cinta de $6,5 \mathrm{~cm}$ de largo por $7 \mathrm{~mm}$ de ancho, que cerramos con un punto de polypropyleno.

Al cabo de dos años, tuvimos dos migraciones de banda en pacientes operados en la primera clínica, y pensamos que podría tener relación con el tipo de material empleado. Al mismo tiempo nos pareció importante conocer si era de utilidad real poner un anillo de restricción en el bypass, por lo que decidimos continuar poniendo anillo sólo en la segunda clínica y no en la primera, en que habíamos detectado migraciones. Operamos doscientos dieciseis pacientes sin instalar anillo (Grupo B). Todos los pacientes tienen consentimiento informado, y todos fueron seguidos por al menos seis años (Figura 2). 


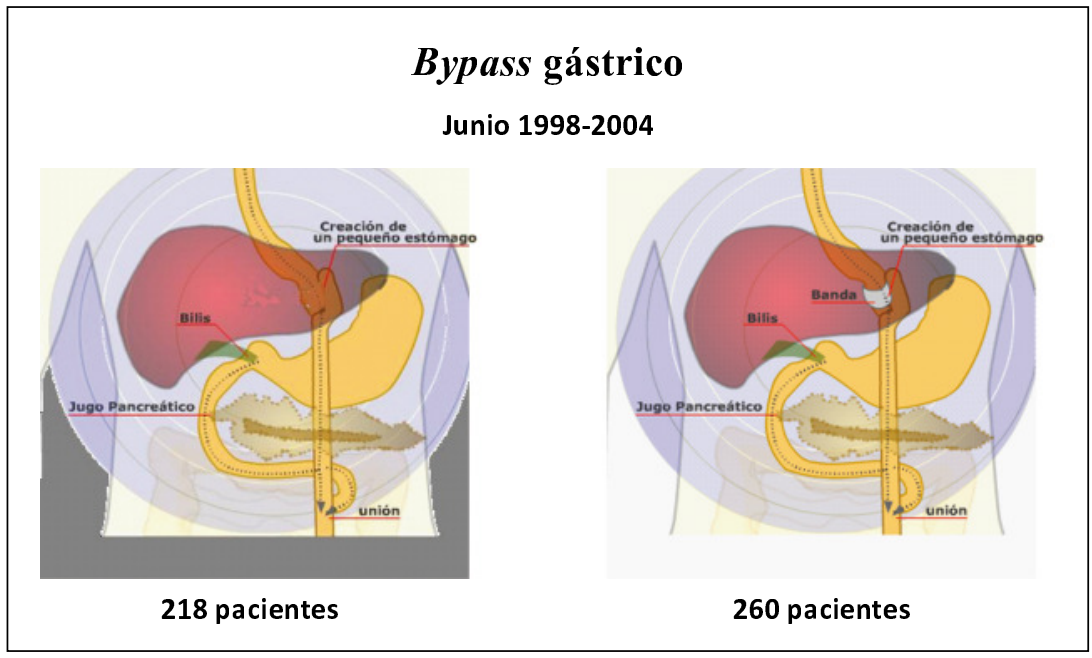

Figura 1. Técnica de Fobi Capella para el bypass con y sin banda. Los dos grupos de pacientes.

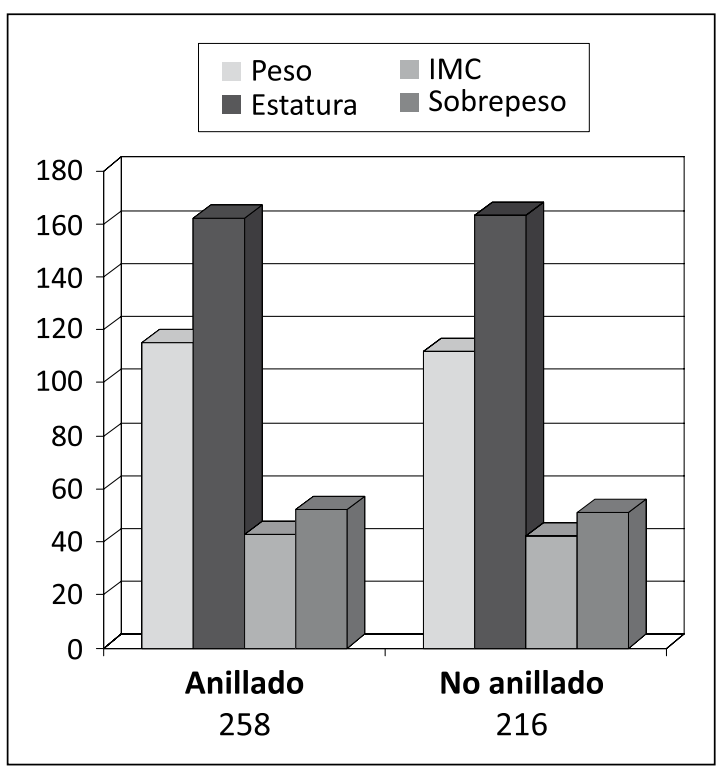

Figura 2. Demografía. Ambos grupos de pacientes son del todo equivalentes.

\section{Técnica quirúrgica}

Todos los pacientes fueron sometidos a un $b y$ pass gástrico por el mismo equipo de cirujanos, con la técnica descrita de Fobi-Capella ${ }^{4,5}$. El 80\% de las cirugías fueron abiertas y el $20 \%$ laparoscópicas. Se prepara un asa desfuncionalizada de $150 \mathrm{~cm}$ de largo con una enteroenteroanastomosis a $50 \mathrm{~cm}$ distal al ligamento de Treitz. Luego creamos un bolsillo gástrico de $20 \mathrm{cc}$. En el grupo A instalamos una banda de $6,5 \mathrm{~cm}$ de largo y $7 \mathrm{~mm}$ de ancho de polyurethane $\left(\right.$ Gore-Tex $\left.{ }^{\circledR}\right)$ a un $\mathrm{cm}$ sobre el borde anastomótico gastroyeyunal, y a través de una apertura en el epiplón menor. Cerramos la banda con un punto de polypropylene 0 , con lo cual queda constituido un anillo de $1,9 \mathrm{~cm}$ de diámetro, alrededor de la pared gástrica. El asa yeyunal es pasada por vía retrocólica y la suturamos al borde vertical de sección del bolsillo de modo que se interpone entre ambas partes del estómago, con una sutura absorbible 3-0. La gastroenteroanastomosis la hacemos amplia de unos $3 \mathrm{~cm}$ de diámetro, con el mismo material de sutura. La banda queda sostenida en su sitio por el asa interpuesta y el ojal en epiplón menor (Figura 1).

En el grupo B el procedimiento es idéntico pero sin poner anillo y además la gastroenteroanastomosis es calibrada a $15 \mathrm{~mm}$ de diámetro.

\section{Seguimiento y controles}

Los pacientes fueron controlados cada mes al comienzo, luego cada tres meses y a partir del tercer año cada seis meses.

La evaluación post-operatoria incluye los siguientes parámetros: \% PEP, usando como peso ideal un IMC de 23; para la calidad de vida se usó el test BAROS y el cuestionario Moorehead-Ardelt modificado. Se prestó especial atención al tema de la tolerancia a la alimentación. También se evaluó la presencia de complicaciones.

\section{Estadísticas}

La casuística se colectó en el LapBase Data System. Usamos el test $t$-de Student, para analizar las diferencias en \% PEP. Empleamos el programa SSP (Smith Statistical Package), que da en forma automática un cuadro en inglés, con los resultados de promedios, desviación standard y valor $\mathrm{P}$ de significancia. 


\section{Resultados}

Encontramos un \% PEP significativamente mayor en el grupo A, desde los 36 meses de post-operatorio y hasta los diez años de seguimiento (Figura 3), con una diferencia de un $19 \%$ entre ambos grupos al término del estudio. La diferencia se incrementa con el tiempo. Los pacientes con banda mantienen su \% PEP hasta los 11 y 12 años. Tenemos pacientes sin anillo sólo hasta 10 años, pues empezamos más tarde a hacer este procedimiento. La CDV es similar en ambos grupos, como mostramos en la Figura 5. En cuanto a las complicaciones relacionadas con la banda, tenemos tres casos de migraciones al interior del estómago, todas con el anillo de PTFE, lo que ocurrió con los pacientes del comienzo del estudio (Figura 4). Con la banda de polyuretano (Gorotex $\left.{ }^{\circledR}\right)$, no hemos observado migraciones ni deslizamientos en más de 10 años. Por el contrario, y en relación a las complicaciones derivadas del anillo, comprobamos que la estenosis de la gastroyeyunoanastomosis es significativamente más frecuente en los bypass $\sin$ anillo, $4 \%$ versus un $0,4 \%$ en los bypass anillados.

En nuestra experiencia, al primer año, la resolución de comorbilidades es similar en ambos grupos, pero al cabo de 10 años hay alguna mayor recurrencia de diabetes y dislipidemia en los pacientes sin anillo y relacionado con re-ganancia de peso, sin embargo, los números son muy escasos para extraer alguna conclusión valedera (Tabla 1 ).

En Tabla 2, mostramos el número de pacientes y el \% de seguimiento. En la Tabla 3 vemos los resultados del Student's $t$-test que compara los Grupos A y B a 3 años y luego de 9 y 10 años.

\section{Discusión}

Hay pocas publicaciones que comparan BPG con BPGA en el largo plazo. En esta comparación es importante evaluar no sólo la diferencia en la baja de peso sino también la calidad de vida, la presencia de vómitos, y tolerancia a la alimentación. Además es fundamental investigar si el anillo produce problemas

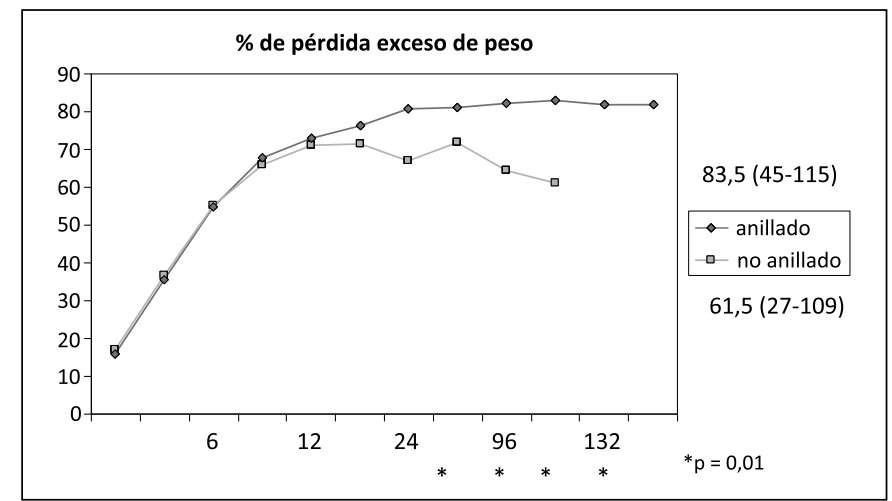

Figura 3. Comparación de \% PEP ambos grupos hasta 10 años.

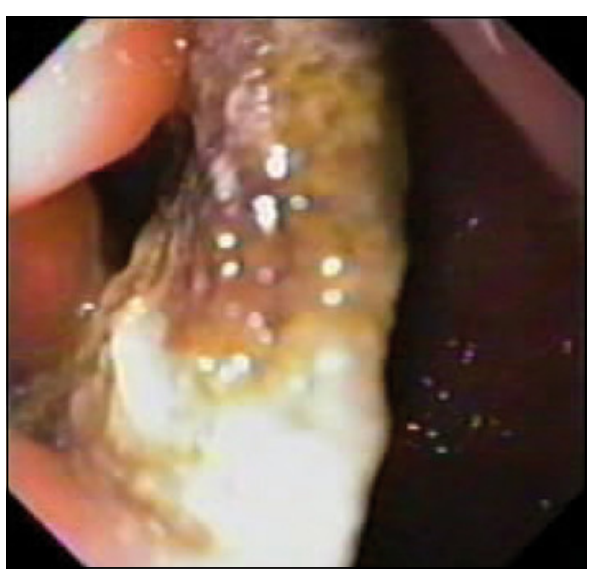

Figura 4. Banda migrada al interior del bolsillo gástrico. Fue extraída por endoscopia.

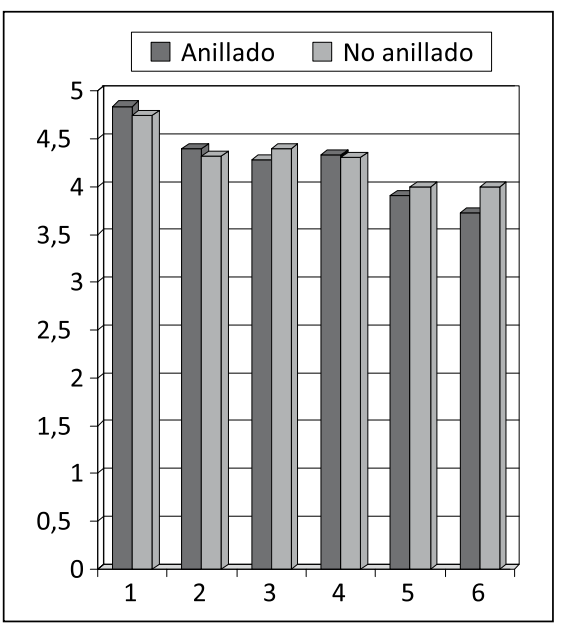

Figura 5. Calidad de vida: de acuerdo a test de Baros y encuesta de Moorehead-Ardnelt. 1) autoestima, 2) actividad física, 3) actividad social, 4) trabajo, 5) sexo, 6) tolerancia a la alimentación.

Tabla 1. Corrección de comorbilidades

\begin{tabular}{|lcccccc|}
\hline A 10 años & \multicolumn{2}{c}{ Diabetes } & \multicolumn{2}{c|}{ Dislipidemia } & \multicolumn{2}{c|}{ Apnea } \\
Anillado & $3 / 4$ & $75 \%$ & $10 / 15$ & $66 \%$ & $3 / 4$ & $75 \%$ \\
No anillado & $2 / 4$ & $50 \%$ & $4 / 8$ & $50 \%$ & $3 / 5$ & $60 \%$ \\
\hline
\end{tabular}


BYPASS ANILLADO ¿CUÁL ES SU REAL UTILIDAD? ESTUDIO COMPARATIVO A DIEZ AÑOS

Tabla 2. Porcentaje de seguimiento en diferentes períodos

\begin{tabular}{|lcccccc|}
\hline & \multicolumn{3}{c}{ Con anillo } & \multicolumn{3}{c|}{ Sin anillo } \\
\hline 1 año & \multicolumn{2}{c}{ Seguimiento } & \% de seguimiento & \multicolumn{2}{c|}{ Seguimiento } & \% de seguimiento \\
3 años & 258 & 244 & 93,85 & 216 & 194 & 88,99 \\
\hline 7 años & 240 & 169 & 69,26 & 192 & 163 & 84,02 \\
\hline 8 años & 148 & 111 & 73,51 & 78 & 56 & 70 \\
\hline 9 años & 94 & 78 & 82,98 & 49 & 41 & 78,85 \\
10 años & 67 & 51 & 75 & 36 & 29 & 78,38 \\
\hline
\end{tabular}

Tabla 3. Test de Student dos variables \% PEP

\begin{tabular}{|lccc|}
\hline & a 3 años & a 9 años & a 10 años \\
Variante 1, número de observaciones & 169 & 51 & 34 \\
Promedio primera muestra & 77 & 82 & 81 \\
Variante 2, número de observaciones & 163 & 29 & 17 \\
Promedio segunda muestra & 72 & 61 & 62 \\
Valor p para dos variables & 0,0174 & 0,000000005 & 0,00008 \\
\hline
\end{tabular}

como el deslizamiento o migración, que pueden hacer dudar de los beneficios de poner un mecanismo de restricción.

De nuestros resultados es claro para nosotros que tenemos mucho mejor \% PEP en el grupo anillado, si los seguimos en el largo plazo. La mayoría de las publicaciones efectuadas por cirujanos que usan banda en el bypass, tiene sólo dos o tres años de seguimiento $^{7-10}$. Hay solamente un estudio hasta ahora que no muestra diferencias entre BPGA y sin anillo, pero el resultado es a dos años de seguimiento ${ }^{11}$. Buchwald, encuentra en un grupo de 4.204 bypass, un $\%$ PEP de $65,1^{1}$ después de dos años de control; Salinas, en un grupo de 3.500 BPGA muestra un $83 \%$ de PEP a cinco años de seguimiento ${ }^{12,13}$. La comparación entre estos dos grupos de pacientes permitió plantear con más claridad que había una diferencia favorable en el \% PEP en los bypass anillados, a pesar de que ambas poblaciones no eran estrictamente comparables, pues en el grupo de revisión de Buchwald había algunos pacientes también anillados. En nuestro grupo de pacientes tenemos alrededor de un $70 \%$ de seguimiento a 10 años, lo que hemos conseguido llamando por teléfono o enviando correos a los pacientes que no han venido a control por algún tiempo, y en algunos casos hemos tenido que confiar en una encuesta telefónica o por correo electrónico. En la Tabla 2, mostramos el número de pacientes y el \% de seguimiento. En la Tabla 3, ve- mos los resultados del Student's $t$-test que compara los Grupos A y B a 3 años y luego de 9 y 10 años; los resultados son muy significativos.

Respecto de la calidad de vida encontramos que no hay diferencias entre los dos grupos, lo que está de acuerdo con otras publicaciones ${ }^{7-12}$. En la encuesta nos preocupamos especialmente de investigar la tolerancia a la alimentación. El grupo A muestra una mayor dificultad a la ingesta, que ocurre especialmente si no mastican bien o ingieren bocados un poco más grandes. Nos parece que este es un hecho deseable, pues les ayuda a mantener el hábito de comer lento y en volúmenes apropiados, lo que es importante en el resultado final del procedimiento. Esta limitación sin embargo, no es apreciada por el paciente como una menor calidad de vida.

Nos parece que es muy importante escoger un buen material para confeccionar el anillo, y también el tamaño adecuado. Se ha usado una gran variedad de materiales, como malla de polypropylene, anillos de silicona, y otros ${ }^{14}$. El anillo debe ser bien tolerado, suave y dúctil, y es mejor que tenga una cierta elasticidad, pues un anillo muy rígido hace más difícil pasar el bolo alimenticio al momento de la ingesta. Se han usado distintas longitudes o diámetros de anillos, entre 4,5 y $7,5 \mathrm{~cm}$ de $\operatorname{largo} 0^{10,13-15}$. Llegamos pronto a la conclusión que $6,5 \mathrm{~cm}$ es un buen largo de anillo, que permite una restricción adecuada sin producir disfagia. Esta banda la cerra- 
mos con un punto no absorbible y queda entonces un anillo de 1,9 $\mathrm{cm}$ de diámetro, alrededor de la pared gástrica, de modo que puede variar el tamaño real del paso dependiendo del grosor de esta pared; se ha encontrado una buena correlación entre el diámetro real, medido por endoscopia y el \% $\mathrm{PEP}^{12}$. La idea de poner anillo no es estrechar el paso del alimento sino evitar que éste se dilate con el tiempo y evitar también que crezca y se dilate la primera porción del asa yeyunal anastomosada al bolsillo gástrico, lo que puede llegar a constituir un gran neo-estómago. Hay interesantes estudios con TC multicorte con contraste que permiten visualizar estos cambios ${ }^{16}$.

Otro aspecto interesante es que el anillo establece el tamaño del paso del alimento, por lo cual no es necesario calibrar la anastomosis, como debe ser en el bypass no anillado. En el bypass anillado podemos hacer una amplia anastomosis sin arriesgar una recuperación del peso. Mientras más calibrada es la anastomosis mayor es la posibilidad de estenosis de la misma ${ }^{9,13,14}$. En nuestra experiencia hemos tenido sólo una estenosis en $900 \mathrm{BPG}$ anillados $(0,11 \%)$ y 8 estenosis en el grupo no anillado (4\%). Es decir, las estenosis son 40 veces más frecuentes en este último grupo. Mali no tiene ninguna estenosis con anastomosis de $4 \mathrm{~cm}$ de amplitud ${ }^{15}$.

Con el anillo de PTFE hemos tenido tres migraciones al interior del estómago, lo cual es una molesta complicación, aunque es fácil de resolver endoscópicamente ${ }^{16-25}$. A raíz de esto hemos dejado de usar este material, y con el parche vascular de Gore-Tex ${ }^{(\mathrm{R})}$ no hemos tenido ni migración ni deslizamiento por más de 10 años. Por esto es muy importante encontrar el correcto tipo de anillo que no migre y sea bien tolerado por el organismo. Actualmente, hay productos especialmente diseñados que han dado excelentes resultados, siendo de más fácil instalación.

En relación a la corrección de comorbilidades podríamos esperar un mejor control en los pacientes que tienen mayor \% PEP en el largo plazo. Sin embargo, no hemos encontrado publicaciones respecto de resolución de comorbilidades relacionadas con este punto. En nuestros pacientes, la resolución de comorbilidades es igual en ambos grupos al año de operados; a los 10 años encontramos una leve mayor recurrencia de diabetes y de dislipidemia en los pacientes sin anillo, sin embargo, los números son muy pequeños para establecer alguna conclusión.

Para nosotros, está claro que tiene importantes ventajas instalar algún mecanismo de restricción en el tracto de vaciamiento del bolsillo gástrico, y usamos un anillo en todos nuestros pacientes operados de bypass. Aquellos que apoyan la instalación de este mecanismo de restricción han sido bastante proactivos en recomendarlo, sin embargo, la mayoría de los cirujanos no están dispuestos a usar una banda de restricción, tal vez porque implica un pequeño tiempo quirúrgico, o un costo, o por temor a usar un cuerpo extraño que puede migrar. Otra posible razón es que no hay seguimientos en el largo plazo. Pero posiblemente la causa más importante es que, si bien los resultados sin la banda son más pobres, no lo son en extremo, lo que hace que la colocación del anillo no sea tan esencial ${ }^{21}$.

\section{Referencias}

1. Buchwald H, Avidor Y, Braunwald E, Jensen MD, Pories W, Fahrbach K, et al. Bariatric surgery: a systematic review and meta-analysis. JAMA 2004;292:172437.

2. Mason EE, Doherty C, Cullen JJ, Scott D, Rodríguez EM, Maher JW. Vertical gastroplasty: evolution of vertical banded gastroplasty. World J Surg.1998;22:91924.

3. Linner JH. Comparative effectiveness of gastric bypass and gastroplasty. Arch Surg. 1998;22:925-35.

4. Fobi MAL, Lee H, Flemming AW. The surgical technique of the banded gastric bypass. J Obes Weight Regul. 1989;8:99-102.

5. Fobi MAL. El presente y futuro de la cirugia bariátrica. Rev Chil Cir. 2009;68:79-82.

6. Capella RF, Capella JF, Mandec H, Nath P. Vertical banded gastroplasty-gastric bypass: preliminary report. Obes Surg. 1991;1:389-95.

7. Awad W, Garay A, Oñate VH, Turu J. Gastric bypass with and without a ring: the effect on weight reduction and quality of life. Obes Surg. 2005;15:724-9.

8. Bessler M, Daud A, Kim T, DiGiorgi M. Prospective randomized trial of banded versus nonbanded gastric bypass for the super obese: early results. Surg Obes Relat Dis. 2007;3:480-4.

9. Awad W, Garay A, Martínez C, Oñate V, Turu I, Yarmuch J. Descenso ponderal y calidad de vida mediante la cirugía de Bypass gástrico con y sin anillo de calibración. Rev Chil Cir. 2008;60:17-21.

10. Valezi AC, Brito EM, Souza JCL, Guariente ALM, Emori FT, Lopes VCH. A importância do anel de silicone na derivação gástrica em Y-de-Roux para o tratamento da obesidade. Rev Col Bras Cir. 2008;35:18-22.

11. Arceo-Olaiz R, Nayví España-Gómez M, MontalvoHernández J, Velázquez-Fernández D, Pantoja J, Herrera MF. Maximal weight loss after banded and unbanded laparoscopic Roux-en-Y gastric bypass: a randomized controlled Trial. SOARD 2008;4:507-11.

12. Salinas A, Salinas BHM, Santiago E, García W, Ferro Q, Antor MM. Silastic ring vertical gastric bypass: cohort study with $83 \%$ rate of 5 -year follow-up. SOARD 2008;5:455-8.

13. Evangelista LF, Campos JM, Ferraz AA, Escalona A, Neto MG, Ramos AC, Ferraz EM. Uso de anillo en 
bypass gástrico: Ventajas y desventajas. Rev Chil Cir. 2009;61:571-7.

14. Stubbs RS, O'Brien L, Jurikova L. What ring size should be used in association with vertical gastric bypass? Obes Surg. 2006;16:1298-303.

15. Mali Jr. J, Fernandes FAMH, Valezi AC, Matsuo T, Menezes MA. Influence of the actual diameter of the gastric pouch outlet in weight loss after silicon ring roux and y gastric bypass: an endoscopic study. Obes Surg. 2010;20:1231-35.

16. Karcz WK, Kuesters S, Marjanovic G, SueBlin D, Kotter D, Thomusch O, et al. 3D-MSCT Gastric Pouch Volumetry in Bariatric Surgery. Preliminary Clinical Results. Obes Surg. 2009;19:508-16.

17. Arasaki CH, Del Grande JC, Yanagita ET, Alves AK, Oliveira DR. Incidence of regurgitation after the banded gastric bypass. Obes Surg. 2005;15:1408-17.

18. Fobi M, Lee H, Igwe D, Felahy B, James E, Stanczyk $\mathrm{M}$, et al. Band erosion: incidence, etiology, management and outcome after banded vertical gastric bypass. Obes Surg. 2001;11:699-707.

19. Rocha LCM, Lima Jr GF, Martins da Costa MEVM, Girundi MG, Farah MW. A Endoscopia em pacientes submetidos à cirurgia de Fobi-Capella - Análise retrospectiva de 800 exames. GED 2004;23:195-204.

20. Galvão Neto MP, Campos JM, Garrido T, Evangelista LF. Migración del anillo después del bypass gástrico. En: Campos JM, Galvão Neto MP, Moura EGH (Org.).
Endoscopia en cirugía de la obesidad. 1a ed. Caracas: AMOLCA. p. 181-190.

21. Campos JM, Galvão Neto MP. Tratamento do deslizamento de anel pós-cirurgia de Fobi-Capella. In: Endoscopia gastrointestinal terapêutica. (Org.) SOBEDSociedade Brasileira de Endoscopia Digestiva. São Paulo: Tecmedd; 2006. p.1143-1147.

22. Berti LV, Garrido Jr AB. Bypass gástrico: É essencial a colocação do anel? In: Castro LP, Savassi-Rocha PR, Rodrigues MAG, editores. Tópicos em Gastro- enterologia: Obesidade e Urgências Gastroenterológicas. Rio de Janeiro: Medsi; 2003. p. 95-108.

23. Campos JM, Costa Júnior AB, Evangelista LFL. Dificultad de vaciamiento gástrico secundário al anillo. En: Campos JM, Galvão Neto MP, Moura EGH. Endoscopia en cirugía de la obesidad. 1a ed. Caracas: AMOLCA. p.203-213.

24. Campos JM, Evangelista LFL, Siqueira LT, Ferreira Filho HÁ, Ferraz AAB, Ferraz EM. Tratamento endoscópico das complicações do anel no bypass gástrico. In: Anais do Segundo Congresso Latinoamericano International Federation Surgery Obesity-IFSO de Cirurgia para la Obesidad, (Org.). Cacun-México: Colegio Mexicano de Cirugía para la Obesidad 2007. p.145.

25. Elias AA, Matielli JD, Oliveira MR, Berti LV, Gabriel J, Szego T, et al. Complicações relacionadas com anel de silicone na operação de Capella-Fobi. Bol Cir Obes. 2001;2:26. 